\title{
A New Fuzzy Algorithm For Improving Quality of Service In Real Time Wireless Sensor Networks
}

\author{
Roghayeh abbasi ${ }^{1}$, Amir Massoud Bidgoli $^{2}$, Mashaalah Abbasi Dezfoli ${ }^{3}$ \\ ${ }^{1}$ Department of computer engineering, Science and Research Branch, Islamic Azad \\ University, Khouzestan,Iran \\ en_rabbasi@yahoo.com \\ ${ }^{2}$ Department of computer engineering, Islamic Azad University, Tehran North Branch \\ ,Tehran,Iran \\ am_bidgoli@iau-tnb.ac.ir \\ ${ }^{3}$ Department of computer engineering, Science and Research Branch, Islamic Azad \\ University, Khouzestan,Iran \\ m.abbasi@khouzestan.srbiau.ac.ir
}

\begin{abstract}
A wireless sensor network comprise a large number of cheap sensors as nodes. These sensors used for data collection and transmition to a central node(sink), are distributed in packed formats in an intended wide area of operation. One of the vital issues in these networks, is their energy constraints which directly affects the network life span. In real time wireless sensor networks (RWSN), not only energy constraints but data delivery time has a vital role in network successful operations as well as data validation. In real time programs, quality of service parameters such as delays must be reduced to a minimum. Reduction of packet losses to increase reliability of data deliveries is another issue in these networks. In this article, a new routing algorithm with fuzzy technique for improving quality of service in RWSN, is presented. The main objectives of this technique are two folds. The first is reduction of energy consumption which results in an increase in the network operations life span and the second is to meet a defined end-to-end delay and hence increasing reliability by reducing number of packet losses considerably. By using different fuzzy parameters and if-then rules, each node can make a decision to choose its next step for routing towards destination (sink). Simulation results show that our proposed new protocol considerably reduces energy usage in the network as well as guaranting the quality of service and noticeable improved performance in comparison with SPEED and THVR.
\end{abstract}

\section{KEYWORDS}

Real time Wireless Sensor Network, Quality of Service, Fuzzy logic, Delay, Routing.

\section{INTRODUCTION}

Wireless sensor networks consists a large number sensor nodes that communicate with each other using wireless links. Each sensor node is able to receive and forward information to the sink. Each sensor node consists of different parts including: sensors, processor unit, energy source and communication unit.[1]

DOI: $10.5121 /$ ijassn.2012.2201 
The sensor nodes is limited in wireless communication and information processing abilities and requiring them to use a routing protocol to pass the data across the network and reach to the sink. This routing process takes time and some nodes may have to queue incoming data and forward them one by one, These delays cause different data packets to take various times to reach the base station, even if they are sent from a single source.[2] One of the major challenges in this type of networks is limited the amount of energy consumption that is directly related to the lifetime of the network.

Some sensor network applications such as intruder detection, medical care, awareness of the fire, nuclear reactor control and identify health, have real-time requirements for the data. In Realtime Wireless Sensor Network, timely delivery of sensory data plays a crucial role in the success of the mission. This means that require low end-to-end delays in data delivery and data is out of date, leading to disastrous effect. In realtime programs the sensor data is valid only for a limited time duration that called deadline. different types of sensory data have different deadlines depending on the dynamics of the sensed environment. Therefore, sensor network applications require delivery of various types of sensory data with different levels of real-time requirements.

Realtime wireless sensor networks, network Quality Of Service. Each program has requirement certion service of network. Every application has certain service requirements from the network, these requirements are defined as its expected Quality of Service (QoS) [21]. Fundamental parameters quality of service in WSNs include throughput, delay, jitter and packet loss rate. Moreover parameters reliability, timeliness, robustness, availability and security are characterized as QOS network requirements and be used to measure the degree of satisfaction of QOS. The use of these networks in different environments, needs for different QOS proportional with work environment. Hence provisioning acceptable QOS for all possible types of traffic is a challenging problem to topological of sensor networks.

In realtime networks data with limited time must be reach in low delay and end-to-end deadline the destination. Therefore sensor network protocols must supports realtime communication with reduce deadline miss ratio (DMR).

On the other hand, due the nature sensor network, resource constraints, low reliability node, dynamic topology sensor networks, real time application in sensor network should not only consider the time constraints, also routing methods must considered path with energy efficient so that the maximum network lifetime.

So far are presented routing protocols for RWSN, with according to particular parameters such as speed, power transmission, two-step neighbor, multi-speed, multi-path and energy try to meet expected quality of service. But these protocols with respect to fixed parameters are considered an issue in ross.

When there is more than one parameter to making decision, there is different ways such as multiplying or intelligently computing for combination of parameters and creation a single parameter. But according to the characteristics of sensor networks is important that this computing done simple and in less time, to exist to use and implement it in a routing protocol. Fuzzy logic is one of ways combining parameters that due the simplicity method it in solving problems, using in this algorithm. In this method combines input parameters is the simplicity, ease and understandable and the help of fuzzy rules can be easily control and adjust the output parameter and efficiently, that in comparison with other methods may work better.

on the other hand, Fuzzy logic, has potential for dealing with conflicting situations and imprecision in data using heuristic human reasoning without needing complex mathematical 
modelling. Accordingly, in this study, we consider for a node, various parameters that have some kind of compromise with each other and using fuzzy variables and the basic rules to give each node a priority. Nodes with highest priority as the next step is selected to send packets to the destination. Energy aware routing protocol in addition to the maximum network lifetime tries path determine to meet end-to-end deadline and highest reliability for sending the package.

The remaining of this paper is structured as follows. Section 2 summarizes the related work. In section 3 we introduct the proposed protocol. Simulation results and evaluation protocols are discussed in section 4. Conclusions and recommendations expressed in section 5.

\section{RELATED WORKS}

In recent years, protocols are presented to meet Quality of service in RWSNs. A survey of routing protocols for RWSNs and their classification is given in [6,5].

Speed protocol is one of the well known realtime routing protocols. The protocol is considered deadline for each packet that this deadline is guaranteed by using an initial velocity to V. the speed $\mathrm{V}$ is defined respect direct distance from source $\mathrm{S}$ until destination $\mathrm{T}$ to deadline required for each package. After this process, each node, a neighbor that guarantees deadline, selecting and delivers the packet to its. If not found a desirable neighbor, nodes can be removed the packet. This protocol ensures a high rate of packet delivery and is scalable as every mechanism works in a localized manner. But it doesn't consider energy metric and reliability and the other hand, provides only one network-wide level of speed, which is not suitable for differentiating various traffic having different deadlines.

Felemban offered multi-path and multi-SPEED Routing Protocol (MMSPEED) [8] that meets the requirements timeliness and reliability. This protocol works on network layer and MAC layer and offers multiple levels of speed and redundancy. If intermediate nodes found a package that delay deadline with current speed is lost, can raise speed level. Each node can have multiple copies of packets to achieve the desired level of reliability will send a group of neighbors in the neighborhood. In [2] with expression this point that MMSpeed dose not consider the remaining energy node, is introduced energy aware routing protocol based MMSpeed (EAMMSpeed).

In [9] THVR routing protocol based on the information two-hop neighbor is presented for realtime wireless sensor networks. Having more information about the one-hop and two-hop neighbor, the system helps to decide routing with more accurate and also has better results in energy consumption and packet delay.

Introduced protocol in [10] the combination of parameters considered MMSpeed and THVR and defines a new level for speed. Then path is selected based on comparison of speed two-hop neighbor with the new level speed. Performance this protocol is beter on energy balance and in deadline miss ratio is similar THVR.

RPAR protocols to achieve minimum latency communication with low energy consumption, use of the routing decisions by adapting the transmission power to dynamically offers. RPAR acts based on theorem inherent trade off between the transmission power and communication delay. In [12] for proceed problem of packet loss, is integrated the concept used in RPAR and THVR and is proposed the method PATH. Whenever PATH cannot find any two-hop neighbor that can provide required velocity, power adaptation scheme will be invoked to discover neighbors that can achieve higher delivery velocity. 
Mohajerzadeh and yaghmaee in [13] with consideration energy and delay parameters, presented routing protocol to find the optimal route with the lowest energy consumption and lowest end-toend delay for real time wireless sensor networks. They have attempted to minimize end-to-end delay by minimizing transmission delay.

The above approaches discussed and routing protocol introduced in [14,15,16] used of fixed metrics and mathematical modeling for finding routing metrics.

in this paper we useing fuzzy logic, various metrics that have tradeoff together such as delay and energy considered and present a efficient algorithm to improve the quality of service RWSN. Fuzzy logic has been successfully applied in various areas including communications and has shown promising results.

\section{FUZZY LOGIC}

With the move towards the information age, human knowledge is very important. Therefore we need the concept of human knowledge to be able toformulate a systematic way it does with other mathematical models in engineering systems.

Fuzzy logic is a new technology for the design and modeling techniques that require a sophisticated mathematics and advanced, Using linguistic values and expert knowledge will be replaced.

Fuzzy sets theory, a theory for action under uncertainty; This theory is capable of many of the concepts and variables and systems that are inaccurate, formulated a mathematical to give And areas for reasoning, inference, control and decision makingunder uncertainty provided.

Fuzzy set theory by Professor Lotfi Zadeh in 1965, Iranian scholarand Master at American University, Berkeley released. Master Lotfi Zadeh After introducing the fuzzy set, fuzzy concepts proposed in 1968, Fuzzy decision making in 1970 and 1971 respectively, the fuzzy presented. In 1973 he built the basis of fuzzy control. In 1975 two scientists fuzzy logic to Mamedani and Asylyan fuzzy control first real design.

Fuzzy systems, the knowledge based systems or rules; The heart of a fuzzy system is a knowledge base of fuzzy rules if he is made.

One of the features use of the legal structure based on fuzzy logic is fuzzy logic During which issues control a set of rules it - then turn into the responders to desired output of the system input is given to the system. This simple rules and obvious to describe the response desired system with the terms of linguistic variables rather than,mathematical formulas are used. Obviously, each fuzzy motors optimization time and processing power of are major issues.

Interesting point that although the fuzzy systems phenomenon of uncertain and does unknown describe However, fuzzy theory is a exact theory.

\section{PROPOSED PROTOCOL}

Quality of service parameters, including delay in realtime programs should be met.of other issues Reduce the number of packets lost so that increases the reliability. On the other hand the most important issue in sensor networks is efficient energy consuming Because energy sensor nodes have limited and has a direct impact on network lifetime. In this paper we present an algorithm that uses fuzzy logic to meet the end-to-end delay, improved 
energy consumption in network and increases lifetime and reliability of the network with minimizing the number of packets lost.

For each data packet created a deadline that packet must arrive to destination in this deadline to meet guarantee quality of service (QOS). For this task should be end-to-end delay no more than the deadline. We assume this algorithm to meet guarantees Software RealTime (SRT) and well algorithm is aware of the location. Namely each node is aware of its geographic location and ID nodes is defined as locally, so that each node only from its neighbors be distinic.

\subsection{PROTOCOL DESCRIBED}

The purpose in this fuzzy algorithm to determine the next hop for the packet forwarding to destination so that end-to-end delay and reliability to provide for send packets and to optimize energy consumption, increase network lifetime.

Periodically each node, a message notifications will exchange with his neighbors and Receives information required from them. Each node sends a message to its neighbors within its radio range, any node that recives the message, ID, its distance to the sink (D), its position, Residual energy (Eresdual) and the delay until the desired node placed in the message and sends for the source node. In this case, the following each node will have the information about its neighboring nodes:

\begin{tabular}{|l|l|l|l|r|r|}
\hline ID & $\mathrm{X}_{\text {pos }}$ & $\mathrm{Y}_{\text {pos }}$ & $\mathrm{D}$ & $\mathrm{E}_{\text {residual }}$ & Delay \\
\hline
\end{tabular}

ID: is a unique number that is assigned to each node.

$\mathrm{X}_{\mathrm{pos}}, \mathrm{Y}_{\mathrm{pos}}$ : to order are the horizontal position and vertical of the neighboring nodes.

Delay: is delay of the neighbor node that is calculate as follows:

$$
\text { Delay }=\text { TIME_RECV }- \text { TIME_SEND }
$$

TIME_RECV: when a message is recived by the node and TIME_SEND: when a message is sent from the source to the current node.

D: distance node to sink is that a node its distance to sink knows well.

Our fuzzy model include five input fuzzy variables and one output fuzzy variables. Figure 1 shows our fuzzy model. Input fuzzy variables are: node residual energy, distance to sink node, delay between two nodes, factor confidence and deadline package.thus fuzzy rules includes $3^{2} *$ $2^{2} * 4=144$ is the law. Figure 2 shows details of the input fuzzy variables. The output variable is called a priority which each node gives priority to be selected as the next step and in DefuzziFication phase, the fuzzy be assigned constant to priority. 
International Journal Of Advanced Smart Sensor Network Systems ( IJASSN ), Vol 2, No.2, April 2012

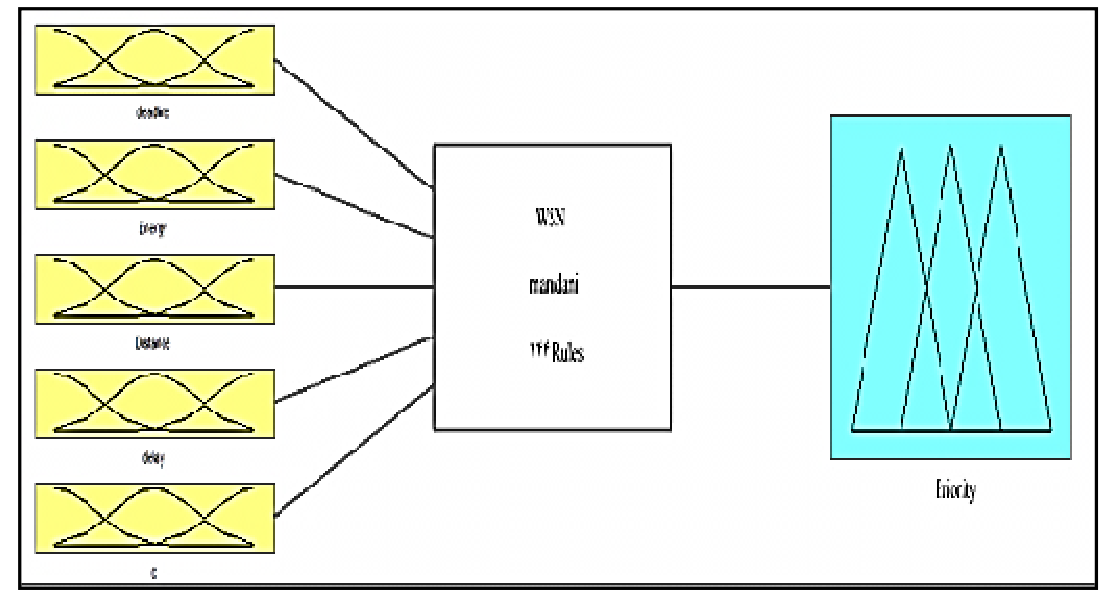

Figure 1. model fuzzy systems

The Node that is less distance and delay, there is a higher priority than other nodes.

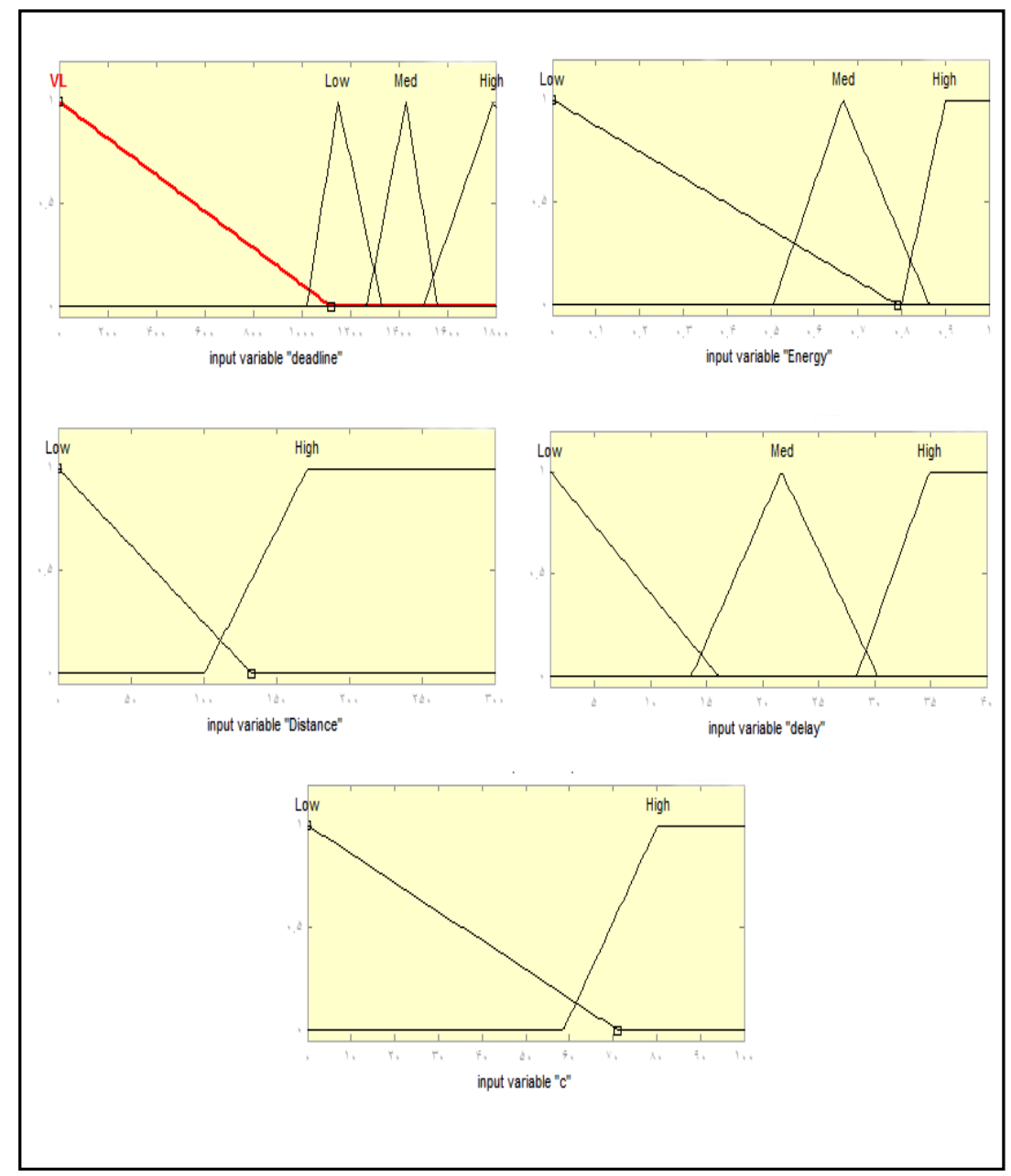

Figure 2. input fuzzy variable 
Confidence factor node (C): is specifies the node reliability that the first is equal 100 or High. This parameter helps in Time Traffic and congestion at a node, to be given less priority. So is increased the reliability arrive package to the destination.

DeadLine Package: to each packets will be dedicated during creation a deadline that must be satisfy. Deadline variable includes 4 membership function. Very Low specifies the deadline is very low and so on Low, Medium, High are specified.

Output variable includes 5 membership function (Very High, High, Medium, Low, Very Low ). Very High defines the node with higher priority. Figure 3 shows the output fuzzy variable. To each node will assign priority between 0 to 100 .

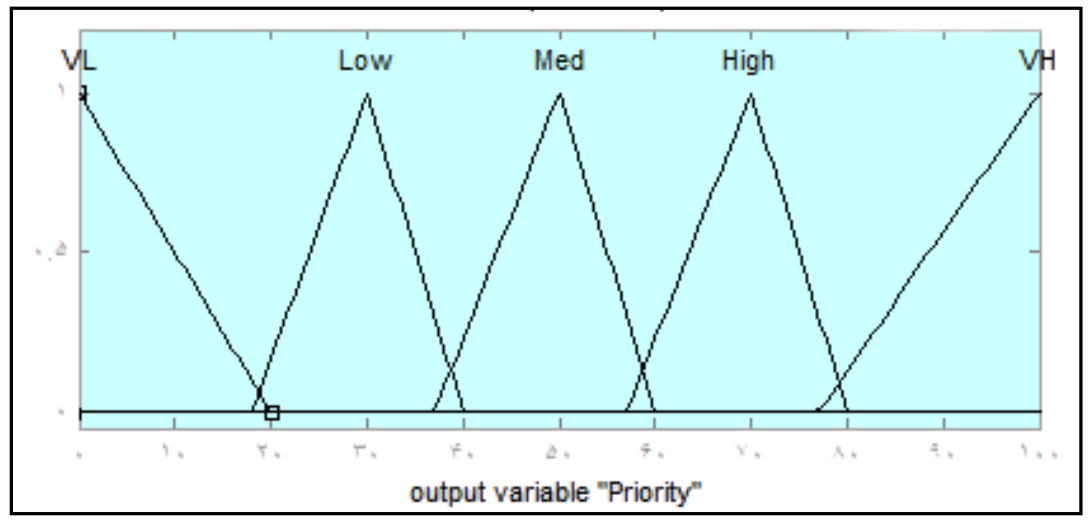

Figure 3. Output fuzzy variable

In this paper we use one of the most common fuzzy inference techniques to name Mamdani Method. This process has four steps:

1. Fuzzification: in this phase, fixed values of the input received for input variables and is determined that the inputs are assigned to each fuzzy set.

2. Evaluation rules: fuzzy input values are received and to using of fuzzy rules are used them. Min method is used at this stage. Some rules are shown in table 1.

1. Output aggregation rules: this process can one output all the rules. In this stage the MAX method is used.

2. Defuzzification: input to this process, the aggregate output fuzzy set, priority, and its output is a costant value. To find a constant output value from the centroid method is used.

Centroid finds equilibrium point in problem fuzzy space with calculated the middle fuzzy. Centroid finds the balance point of the solution fuzzy region by calculating the weighted mean of the fuzzy region. Mathematically, the crisp output domain value R, from solution fuzzy region A, is given by:

$$
\mathrm{R}=\sum_{i=0}^{n} w_{\mathrm{i}} \mu_{\mathrm{A}}\left(\mathrm{W}_{\mathrm{i}}\right) / \sum_{i=0}^{n} \mu_{\mathrm{A}}\left(\mathrm{W}_{\mathrm{i}}\right)
$$

$\mathrm{W}_{\mathrm{i}}$ is the domain value corresponding to rule $\mathrm{i}, \mathrm{n}$ is the number of rules triggered in the fuzzy inference engine and $\mathrm{A}(\mathrm{Wi})$ is the predicate truth for that domain value. 


\subsection{How to send packets}

When a node receives a packet to send,deadline remaining (DLR) packageobtains:

$$
\begin{aligned}
& \text { Time }=\text { TIME_RECV }- \text { TIME_SEND } \\
& \text { DLR }=\text { DeadLine }- \text { Time }
\end{aligned}
$$

The deadline variables and other input variables to the fuzzy system is given, Fuzzy output node, the minimum distance to the sink, minimum latency, highest reliability and the highest energy As the next step and sends the packet to that node.

Tabel1. Fuzzy Rules

\begin{tabular}{|c|c|c|c|c|c|}
\hline C & Delay $_{(\text {i.j }}$ & D & E $_{\text {residual }}$ & DeadLine & Priority \\
\hline High & Low & L & High & VL & High \\
\hline High & Low & H & High & VL & VL \\
\hline Low & Low & L & High & VL & Low \\
\hline High & Low & L & Med & VL & VH \\
\hline High & Low & H & Med & Low & Low \\
\hline Low & Low & H & Med & Low & VL \\
\hline High & High & H & High & Low & VL \\
\hline High & High & H & High & Med & Low \\
\hline High & High & H & High & High & VH \\
\hline High & High & L & High & Low & Med \\
\hline High & High & L & High & Med & High \\
\hline High & Low & L & Med & Low & High \\
\hline High & Low & L & Low & Low & Low \\
\hline High & Low & L & Low & M & Low \\
\hline Low & Low & L & Low & VL & VL \\
\hline Low & High & L & Med & Low & Low \\
\hline High & Med & L & Med & Low & Med \\
\hline High & Med & L & Med & VL & High \\
\hline High & Med & H & Med & Low & VL \\
\hline High & Med & H & Med & High & VH \\
\hline
\end{tabular}

Each node that receives packets from neighboring nodes, After the send it to the next step, the source node sends an ack TIME variable based authentication packet and puts the message ack. Then the queuing delay time for packets is considered.

\begin{tabular}{|c|c|c|}
\hline ID & E residual & Time \\
\hline
\end{tabular}

Based on the time interval between two nodes factor confidence can be calculated. First, the variable Time, the time limits pecified Then compared to the previous value of $\mathrm{C}$ is reduced. Ranged Table 2 shows the percentage loss of confidence. The new value of $\mathrm{c}$ is calculated as follows:

$$
\mathrm{C}=\mathrm{c}-(\mathrm{c} \times \mathrm{X})
$$


Fact that $\mathrm{X}$ percent of the value of $\mathrm{c}$ is reduced.

Table2. calculation factor confidence

\begin{tabular}{|c|c|}
\hline Time Renge & $\begin{array}{c}\text { Percent Reduction } \\
\text { Confidence }(\mathrm{X})\end{array}$ \\
\hline $5-1$ & 0 \\
\hline $5-10$ & 0.01 \\
\hline $10-20$ & 0.02 \\
\hline $20-30$ & 0.03 \\
\hline $30-40$ & 0.04 \\
\hline $40-50$ & 0.05 \\
\hline $50-60$ & 0.07 \\
\hline $60-70$ & 0.09 \\
\hline $70-80$ & 0.11 \\
\hline Time $>90$ & 0.15 \\
\hline
\end{tabular}

Process until the packet the destination (Sink), it continues.

This protocol, a routing protocol's decision is a step by step, Because each node based on his information takes routing decisions. This feature it enables for larger sensor networks to be scalable.

\section{Simulation}

In this section, using computer simulation of the protocol Fuzzy proposed with the protocol, Speed, are evaluated. Before the performance, we describe the simulation environment. The simulation results and analysis performance of the proposed protocol are compared with Speed and THVR protocols.

\subsection{Simulation Environment}

To simulate the proposed algorithm have used of matlab software. This software with simple implement fuzzy algorithm, to helps us in better simulate. Details of the simulation are shown in table 3.

Table3. Simulation settings

\begin{tabular}{|c|c|}
\hline Radio Range & $40 \mathrm{~m}$ \\
\hline Number Of Node & 100 \\
\hline Network Area & $200 * 200$ \\
\hline Packet Size & $50 \mathrm{byte}$ \\
\hline DeadLine Range & $900 \mathrm{~ms}-1800 \mathrm{~ms}$ \\
\hline $\begin{array}{c}\text { Delay Range } \\
\text { Between Two Node }\end{array}$ & $1 \mathrm{~ms}-40 \mathrm{~ms}$ \\
\hline $\begin{array}{c}\text { Distance From } \\
\text { Sink }\end{array}$ & $0-300 \mathrm{~m}$ \\
\hline Energy a node & $1 \mathrm{joules}$ \\
\hline Elec $_{\text {emp }}$ & $50 \mathrm{nj} / \mathrm{bit}$ \\
\hline $\boldsymbol{\varepsilon}_{\mathrm{emp}}$ & $100 \mathrm{jj} / \mathrm{bit} / \mathrm{m}^{2}$ \\
\hline
\end{tabular}


To compare the performance of protocols, we implemented the proposed protocol as well as the speed and THVR protocol in the same environment. For the simulated network, consists of 100 fixed sensor nodes which are randomly distributed in a $200 \mathrm{~m} * 200 \mathrm{~m}$ area. The source send data is located at area $(180 \mathrm{~m}, 180 \mathrm{~m})$ and sink node is at $(0,0)$.

The energy that a node in each transfer loses, equal is to the energy transfer and energy received package. We use of model in [20] introduced for the energy consumption nodes. K-bit data transmission energy is:

$$
\mathrm{E}_{\mathrm{TX}}(\mathrm{K}, \mathrm{d})=\mathrm{E}_{\text {elect }} * \mathrm{~K} *+\varepsilon_{\mathrm{emp}} * \mathrm{k} * \mathrm{~d}^{2}
$$

$\mathrm{d}$ is distance from the node with the next step, $\mathrm{E}_{\text {elec }}=50 \mathrm{nj} / \mathrm{bit}$ and $\boldsymbol{\varepsilon}_{\text {emp }}=100 \mathrm{pj} / \mathrm{bit} / \mathrm{m}^{2}$. $\mathrm{k}$-bit data the energy received is equal to:

$$
\mathrm{E}_{\mathrm{RX}}(\mathrm{K})=\mathrm{E}_{\text {elect }} * \mathrm{~K}
$$

\subsection{Results Analyze}

At first performance of both protocols are compared at different deadline. Data Miss ratio (DMR) for real-time systems is an important parameter and by dividing the number of packages that successfully reached their destination on the total number of packets sent is obtained.

Figure 4 shows in protocols with increases deadline, DMR reduced. Because More time and more convenient routes the packets to reach their destination.

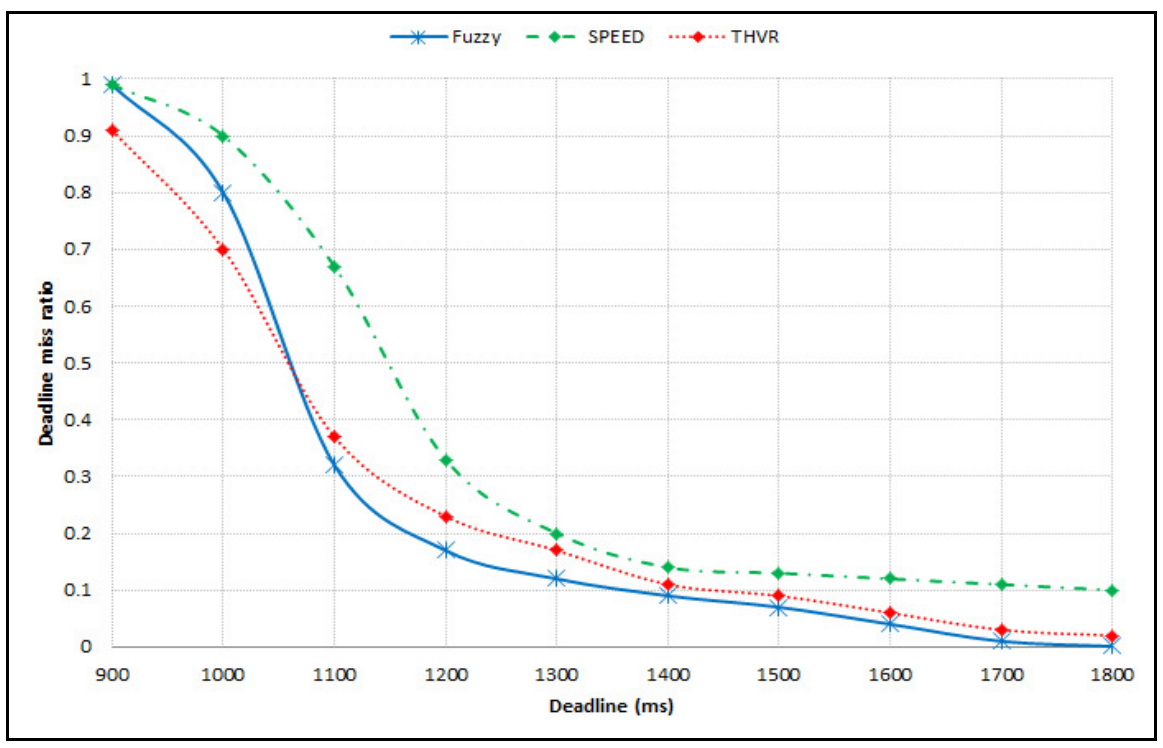

Figure 4. DMR with different DeadLine

The main advantage of fuzzy even at times higher than Speed and THVR, reduce the number of packets is lost. Also in the deadline stringent (e.g. less than 1200ms) this advantage is visible. This is due to consider different measures of distance, delay and factor confidence occurs that Using fuzzy logic the node is selected with minimum distance to the destination and high reliability and leading to decrease the loss of sensitive packets to be delayed. Therefore delay tolerance better fuzzy shows than Speed and THVR. 
Then protocol Fuzzy, Speed and THVR have compare in term of energy consumption. figure 5 shows the energy consumed per packet successfully transmitted.

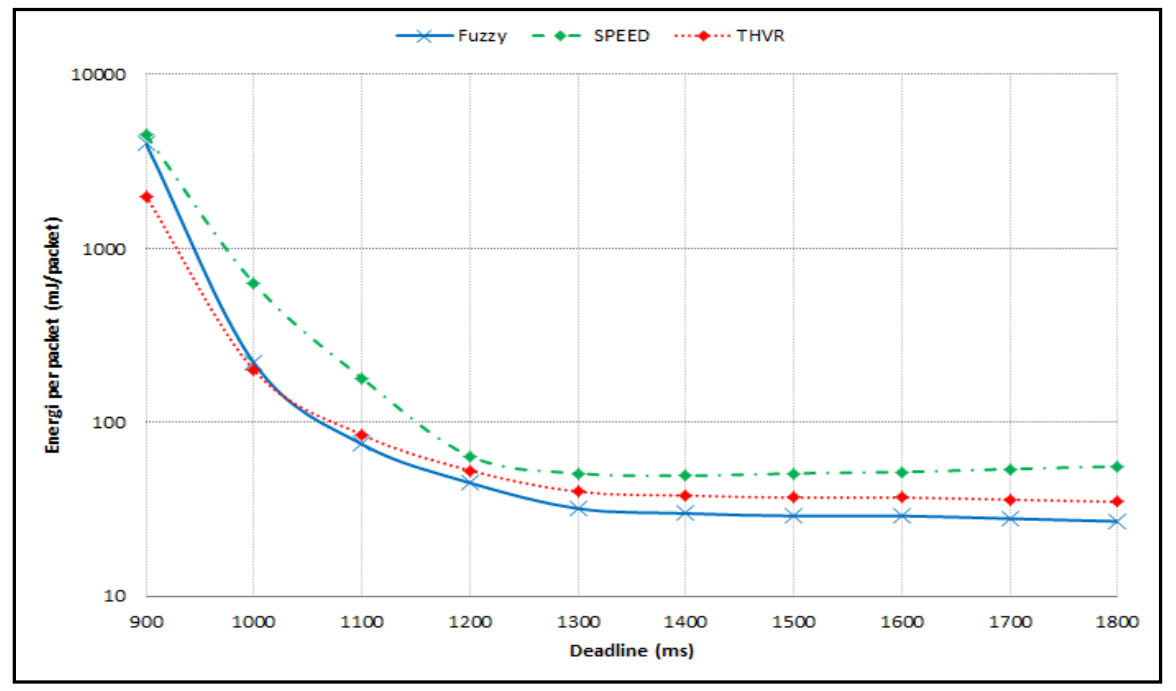

Figure 5. Energy consumed per packet successfully transmitted.

The energy consumption has similar DMR. When a high tolerance of packet delay (e.g., deadline larger than $1400 \mathrm{~ms}$ ), the number of packets successfully transmitted is more and improved stable algorithm. This supports the convergence of overall energy consumption and reduce its. Clearly, our algorithm is more energy efficient than the other two protocols. One of the major reasons is that Fuzzy has a better capability in find a path with small delay.

Furthermore, we investigate the performance of Fuzzy under different workload. Figure 6 shows the DMR in which the number of sources increases from 1 to 6 . the deadline is considered fixed and at $1200 \mathrm{~ms}$.

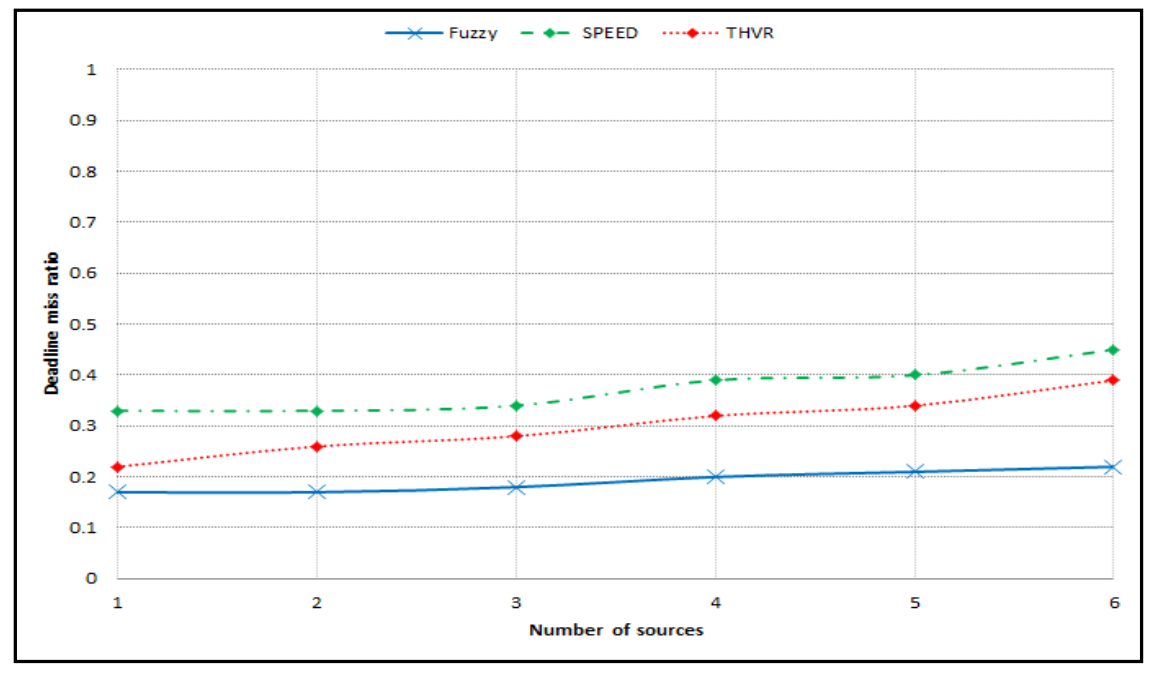

Figure 6. Performance protocols Speed, THVR and Fuzzy while the number of source nodes increases from 1 to 6 . 
Figure 7 shows the energy consumption performance of the three protocols with different source. It is clear that as the number of sources increases, both the DMR and energy consumption

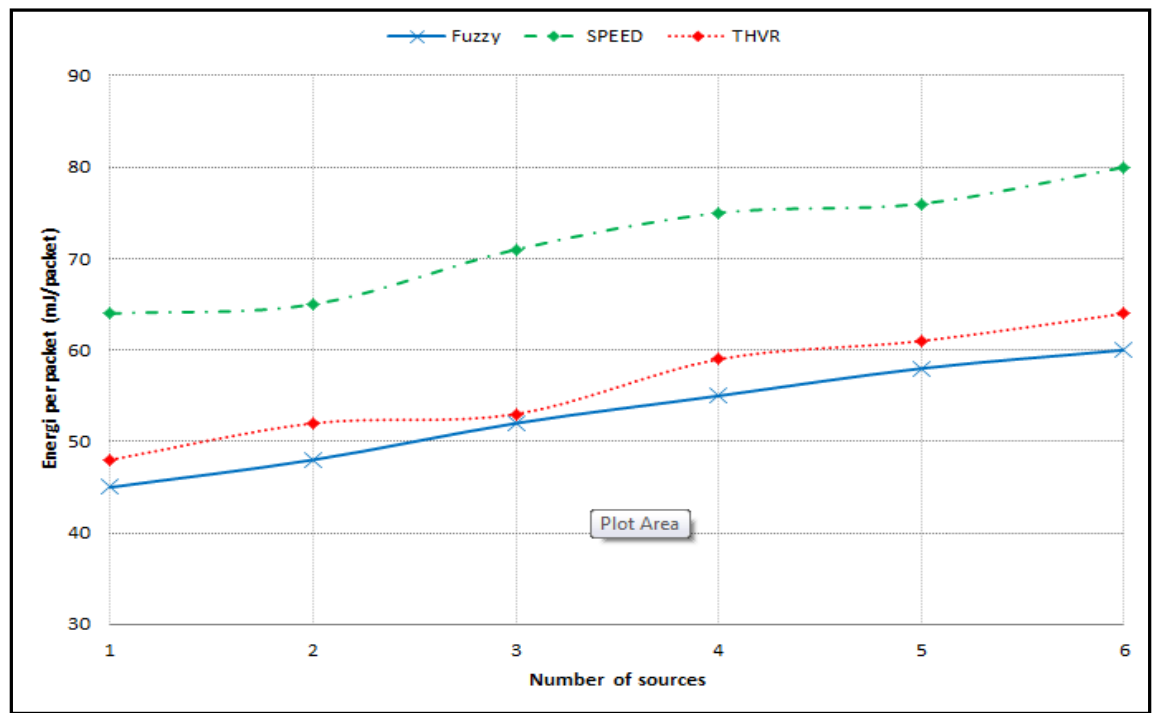

generally increase.

Figure 7. Performance protocols Speed, THVR and Fuzzy in Energy consumed per successfully transmitted packet with different sources.

The increase in DMR is resulted by the increased packet collisions and network congestion. The comparison indicates that Fuzzy has lower DMR and also lower energy consumption per successfully transmitted packe to destination, at sources varied.

Due each node has its factor confidence, if node is density or any reason not to send the packet, lower priority in next run the node is selected.on the other hand, fuzzy logic is fast algorithm. So can be the running time of less than Speed and THVR and of the DMR will perform better.

With sending 80 packets per run, according to the results, the first node in fuzzy algorithm dies after of round 180, while in the Speed after round 100 the first node is lost and this represents the overall energy balance is network. Therefore the protocol fuzzy proposed due to lower energy consumption and better energy balance in the network, increases network lifetime.

Fuzzy Average running time per node is equal to $60 \mathrm{~ms}$, while the Speed average running time in each node is $80 \mathrm{~ms}$, Therefore acts fuzzy about $70 \%$ better.

We claim that this algorithm will perform better with time, because the reliability is closer to the actual value and also can be expressed due of the loss most packets is expired deadline.

\section{CONCLUSION}

We at this research have introduced based on fuzzy algorithm for improving quality of service, that with considering all the parameters such as delay, energy, distance, reliability and deadline packet, Has been able to reduce the rate of data loss And energy distribution is more efficient. Fuzzy approach at comparison with Speed and THVR protocols better results obtained in DMR and energy consumption. On the other hand, due to low computational overhead fuzzy logic, this algorithm is about $70 \%$ of running faster than speed. 
We intend in future algorithms for networks with mobile nodes And different levels of energy for the nodes, we will review.

\section{REFERENCES}

[1] Akyildiz .I. F, Su.W, Sankarasubramaniam. Y, Cayirci. E. 2002 . "A survey on sensor networks". Elsevier Computer Networks, vol 38, pp. 393-422.

[2] Sanati.S, Yaghmaee.M.H, Beheshti.A. 2009."Energy Aware Multi-path and Multi-SPEED Routing Protocol in WirelessSensor Networks", Proceedings of the 14th International CSI Computer Conference (CSICC'09), 978-1-4244-4262-1/09/\$25.00 @2009 IEEE.

[3] RAJSHREE S. DUBEY, RAJNISH CHOUBEY, AMIT DUBEY. 2010. " Challenges for Quality of Service (QoS) in Wireless Sensor Networks". International Journal of Engineering Science and Technology, Vol. 2 (12). 7395-7400.

[4] Xia.f. 2008. "QoS Challenges and Opportunities in Wireless Sensor/ Actuator Networks", Diversity Preservation International (MDPI), Vol. 8, pp. 1099-1110. February 2008.

[5] ZHAN An-dong, XU Tian-yin, CHEN Gui-hai, YE Bao-liu, LU Sang-lu. 2007. "A Survey on Realtime Routing Protocols for Wireless Sensor Networks", State Key Lab. for Novel Software and Technology, Nanjing University Nanjing, Jiangsu 210093, P.R. China.

[6] Sandhya Bansal, Dr Dimple Juneja, Dr Saurabh Mukherjee.2011. "An Analysis of Real Time Routing Protocols for Wireless Sensor Networks". Sandhya Bansal et al. / International Journal of Engineering Science and Technology (IJEST). ISSN : 0975-5462, Vol. 3 No. 3 Mar 2011.

[7] HE T, STANKOVIC J and LU C and Abdelzaher T, 2003, "SPEED: A stateless protocol for real-time communication in sensor networks," in the Proceedings of International Conference on Distributed Computing Systems [C], Providence, RI.

[8] FELEMBAN.E, LEE.C, EKICI E. 2006. "MMSPEED: Multipath multi-SPEED protocol for QoS guarantee of reliability and timeliness in wireless sensornetworks", IEEE Transactions on Mobile Computing [J], 5(6), pp. 738-754.

[9] Yanjun Li, Chang shue, Member IEEE, Ye-Qiong Song, Zhi Wang, Member, IEEE, and Touxian Sun. 2009. "Enhancing Real-tTime Delivery in Wireless Sensor Networks With Two-Hop Information" IEEE Transactions on industrial information, VOL.5, NO.2, MAY 2009.

[10] khalilnejad.M, Ghasemzadeh.M, gha Saram.M.2010. "New Combination Timeliness Routing Porotocol Over Wireless Sensor Networks". World Applied Sciencens Journal 9(7):819-825, ISSN 1818-4952, OIDOSI Publications.

[11] CHIPARA.O, HE.Z, XING.G et al. 2006. "Real-time Power-Aware Routing in Sensor Networks". in the Proceedings of the 14th IEEE International Workshop on Quality of Service (IWQoS 2006) [C], New Haven, CT.

[12] Rezayat.P, Mahdavi.M, Ghasemzadeh.M, Agha Sarram.M. 2010. "A Novel Real-Time Power Aware Routing Protocol in Wireless Sensor Network”. IJCSNS International Journal of Computer Science and Network Security. VOL.10 No.4, April 2010.

[13] Yaghmaee.M.H, Mohajerzadeh.A.H. 2009. "An Efficient Energy Aware Routing Protocol For Real Time Traffic in Wireless Sensor Networks", In IEEE 2009.

[14] Guowei Wu, Chi Lin, Feng Xia, Lin Yao, He Zhang, Bing Liu. 2010. ” Dynamical Jumping RealTime Fault-Tolerant Routing Protocol for Wireless Sensor Networks". journal sensors, ISSN 14248220, March 2010.

[15] Krogmann.M, Heidrich.M, Bichler.D, Barisic.D, Guido Stromberg, "Reliable, Real-Time Routing in Wireless Sensor andActuator Networks", International Scholarly Research Network ISRN Communications and Networking Volume 2011, Article ID 943504, 8 pages.

[16] AKKAYA K, YOUNIS M. 2003."An Energy-Aware QoS Routing Protocol for Wireless Sensor Networks". in the Proceedings of the IEEE Workshop on Mobile and Wireless Networks (MWN2003) [C], Providence, Rhode Island.

[17] Zadeh, L.A. 1965. "Fuzzy sets". Information and Control 8 (3): 338-353. doi:10.1016/S00199958(65)90241-X. ISSN 0019-9958.

[18] Zadeh, L.A. 1968. "Fuzzy algorithms". Information and Control 12 (2): 94-102. doi:10.1016/S00199958(68)90211-8. ISSN 0019-9958.

[19] www.mathworks.com/products/matlab/ 
[20] Heinzelman.W.B, Chandrakasan. A.P, Balakrishnan.H. 2002. "An application-Specific Protocol Architecture for Wireless Microsensor Networks". IEEE Transaction on Wireless Communications Volume 1,pp.660-670 .October 2002.

[21] P. Mohapatra, J. Li, and C. Gui. QoS in mobile ad hoc networks. Special Issue on QoS in NextGeneration Wireless Multimedia Communications Systems in IEEE Wireless Communications, 10(3):44-57, June, 2002.

\section{Authors}

Roghayeh Abbasi received B.Sc. in Computer Engineering, Islamic Azad University Behbahan Branch, Iran in 2008. Since 2009 she has studied the M.Sc in Computer Engineering, Science and Research Branch, Islamic Azad University, Khouzestan, Iran. Her research interests include, Sensor networks, routing, QoS , simulation of networks and wireless network in general.

Amir Massoud Bidgoli is a professor of Computer Engineering in the Department of computer engineering, Islamic Azad University, Tehran North Branch. He received his B.Sc. degree in Electronics Engineering from Lancaster university(UK) and the M.Sc. degree in Computer \& Control Engineering from Salford univercity(UK), in 1987 and 1989, respectively. He received his Ph.D. in Computer Science from Salford\& Manchester University(UK), in 1996.He is a member of IEEE and is indexed in IEEE Explorer. His research interests include computer networks \& evolutionary algorithms, computer architecture, networking, distributed systems and

image processing. He has over fifty publications in journals and ISI conferences

Mashaalah Abbasi Dezfoli is a professor of Computer Engineering in the Department of computer engineering, Islamic Azad University, Science and Research Branch,Khouzestan, Iran.
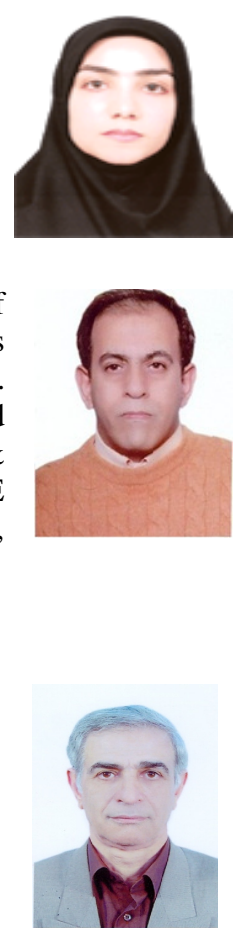